\title{
Classifying Druggability on Potential Binding Sites of Glycogen Synthase Kinase-3 $\beta$ : An In-Silico Assessment
}

\author{
Navneet Chauhan", Anuradha Gajjar² \\ 'Department of Pharmaceutical Chemistry, Institute of Pharmacy, Nirma University, Ahmedabad 382 481, India. \\ 'Department of Pharmaceutical Chemistry and Analysis, Ramanbhai Patel College of Pharmacy, Charotar University of \\ Science and Technology, Changa 388 421, India.
}

\begin{abstract}
Putative binding sites of glycogen synthase kinase- $3 \beta(\mathrm{GSK}-3 \beta)$ have been identified by various computational methods; however, the druggability of these pockets is still unknown. Herein, we assessed a dataset of 24 Protein Data Bank (PDB) crystal structures of GSK- $3 \beta$ using SiteMap to compute the druggability of each identified site. The binding sites were assessed with two site-scoring functions known as the Druggability score (Dscore) and SiteScore (SScore) within SiteMap. An average of eight surface pockets were identified, of which pocket 1 (orthosteric site) and pocket 7 (allosteric site) exhibited ligand-binding characteristics, as analyzed by SiteScore. We further analyzed the druggability of each site with Dscore; pocket 1 proved to be a druggable site, and pocket 7 failed to meet the druggability criteria. The quantitative pocket properties of site 7 were further evaluated to identify plausible reasons for classification as a "difficult" site. In conclusion, these results accurately classified binding sites of GSK- $3 \beta$.
\end{abstract}

Keywords: Allosteric sites, Binding sites; Druggability; Glycogen synthase kinase- 3 ; In silico

\section{INTRODUCTION}

Glycogen synthase kinase-3 (GSK-3) is a serine/threonine protein kinase that exists in two highly homologous forms: GSK-3 $\alpha$ and GSK-3 $\beta$ encoded by two different genes $^{1-3}$. Moreover, in humans, a splice variant (GSK-3ß2) has been reported ${ }^{4}$. The role of GSK-3 as a drug target has been implicated in a variety of unmet human diseases, such as Alzheimer's disease (AD) ${ }^{5-8}$, bipolar disorder ${ }^{9,10}$, various forms of cancers ${ }^{11,12}$, diabetes ${ }^{13}$, and many other diseases ${ }^{14-17}$.

*Corresponding Author: Navneet Chauhan

E-mail address: navneetscientific@gmail.com 
Over the past years "harder targets" that belong to large super-families like kinases have been addressed in drug discovery. Drug modulators that target the kinase catalytic domain risk serious off-target effects ${ }^{18}$. Moreover, it is still challenging to identify other well defined druggable sites on kinases. Kinetic experiments can depict the mode of inhibition but fail to predict the binding locations on the protein; however, pocket detection algorithms can predict likely binding locations of both orthosteric and allosteric pockets. In this research, the GSK-3 $\beta$ binding sites were assessed with a druggability assessment tool (SiteMap) ${ }^{19}$ from Schrödinger, Inc. SiteMap is an energy-based pocket detection algorithm that finds, visualizes, and evaluates protein binding sites. Energy-based methods identify binding sites by docking small organic probes, typically methane or water molecules, on a given target protein to evaluate whether a given protein region interacts favorably ${ }^{20}$. SiteMap is computationally more demanding as this tool provides insights into the physical basis of druggability classification of the target protein sites with high predictive ability.

The X-ray crystal structures reveal three well-known binding sites of GSK-3 $\beta$ : (i) the ATP site, (ii) the substrate binding site, and (iii) the Axin/Fratide binding site. In addition, four allosteric pockets on GSK- $3 \beta$ were recently reported (pockets

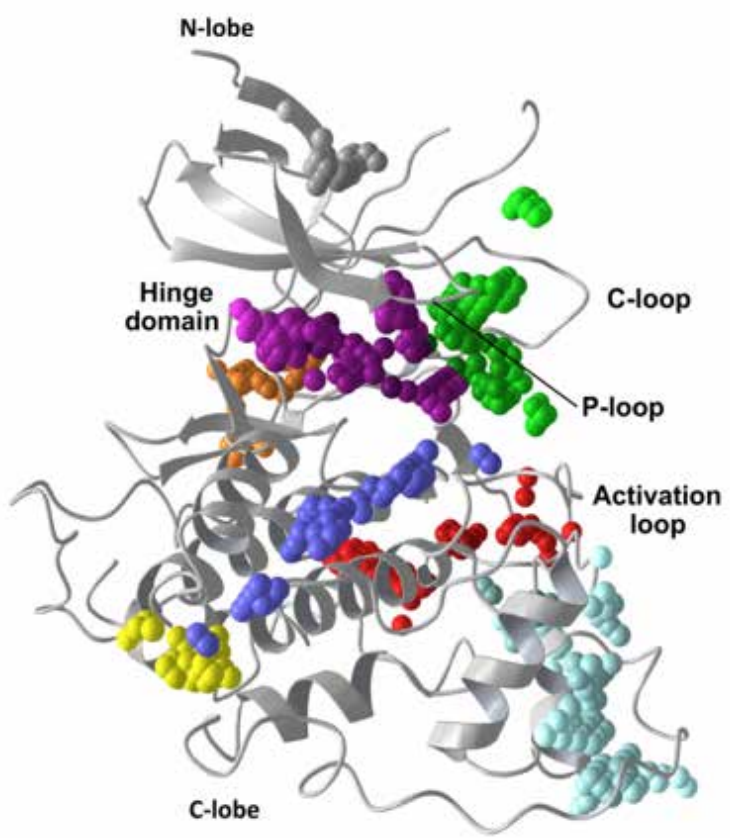

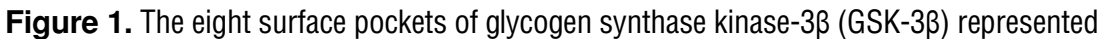
as colored spheres identified by SiteMap [Protein Data Bank (PDB) ID: 4NU1; gray cartoon representation]: pocket 1 (pink spheres), pocket 2 (green spheres), pocket 3 (turquoise spheres), pocket 4 (blue spheres), pocket 5 (gray spheres), pocket 6 (brown spheres), pocket 7 (red spheres), and pocket 8 (yellow spheres). 
4-7) $)^{21}$. Our recent program identified an additional small allosteric pocket at the C-lobe of GSK-3 $\beta$ (pocket 8). Structurally, GSK-3 is a two-domain kinase fold comprising a $\beta$-strand domain and an $\alpha$-helix domain. The residues forming the ATP-binding site (pocket 1 ) are seated deep between the interface of the $\alpha$-helix and $\beta$-strand domains surrounded by a hinge region and a glycine-rich loop, which is often referred to as "P-loop" ${ }^{22-25}$. The substrate binding site (pocket 2) is surrounded by the C-loop and the activation loop. Both pockets are sandwiched between the interfaces of the $\mathrm{N}$ - and C-lobes of GSK-3 $\beta$. The third known pocket is the Axin/Fratide binding site (pocket 3). Pockets 4-8 are the allosteric sites of the kinase shown in Figure 1.

\section{METHODOLOGY}

All computational and molecular modeling studies were carried out using Schrödinger molecular modeling software, version 9.3.5 on a windows Dell workstation.

\section{Protein preparation}

Twenty-four different X-ray crystal structures of human GSK-3 $\beta$ were extracted from the Protein Data Bank (PDB) by maintaining selection criteria close to 2.5 angstroms. GSK- $3 \beta$ is crystallized as a homodimer comprising of chains A and B. In a few cases of the crystallographic structures, chain A monomers were crystallized with Axin and Fratide peptides, and in some cases they exist as a homodimer or monomer protein. The dimer structures within each PDB case were separated to retain the chain A monomers as in many cases, site search may generate unphysical sites over the entire dimer structures. Each individual chain A monomers were preprocessed with the Protein Preparation Wizard in the Maestro program, with the following default options selected: "assign bond orders," "add hydrogen atoms," "create zero-order bonds to metals," "create disulphide bonds," and "delete water molecules beyond $5 \AA$ from heteroatom groups.” The optimal protonation states of each ionizable residue were assigned and the hydrogen-bonding network was optimized for proteins with structural ambiguity. A restrained minimization with an OPLS2005 force field was performed to attain the relaxed state of each refined complex.

\section{Site identification}

SiteMap identifies a site as an enclosed region on the protein surface comprising at least 15 site points (default settings) ${ }^{26}$. To locate a site the search uses a grid of points called "site points." Following the default protocol in SiteMap v2.6, a more restrictive definition of hydrophobicity, a standard grid, and the OPLS2005 force field were used. 
The refined chain A monomers were then submitted to SiteMap for druggability assessment. To avoid a biased search, all crystallographic water molecules, ligands, metal ions, heteroatom groups, unwanted chains, and peptides were removed prior to the site search. SiteMap was assigned to report up to 10 sites to avoid ambiguity that matches with the known sites of GSK-3 $\beta$ (default settings: 5 ).

\section{Druggability assessment by SiteMap}

The druggability of a protein binding pocket is calculated by various physical descriptors in SiteMap, which were calibrated for submicromolar tight-binding sites $^{26}$. These include; (i) the size of the binding pocket and (ii) the volume of a protein site. The above terms are case sensitive and were not calibrated. The enclosure property (iii) indicates the degree to which a site is sheltered from the solvent (calibrated scores: 0.76). The exposure property (iv) measures the degree of exposure to solvent (calibrated scores $\leq 0.49$ ). The degree of contact (v) measures the relative tightness between the site points and the protein site via van der Walls non-bonding interactions (calibrated scores: 1.0). The phobic/ philic character (vi) is a measure of the relative hydrophobic and hydrophilic nature of the site (calibrated scores: 1.0). The balance term (vii) indicates the ratio of hydrophobic to hydrophilic character of the site (the calibrated ratio of the two scores is approximately 1.6). The donor/acceptor character (vii) measures the hydrogen-bonding possibility between a ligand and protein site where a ligand donates and the protein accepts hydrogen bonds within a site (calibrated scores: $\sim 0.76$ ).

SiteMap computes two output scores for each binding site known as the SiteScore (SScore) and the Druggability score (Dscore). Both scores are defined as:

$$
\begin{aligned}
& \text { SiteScore }=0.0733 n^{1 / 2}+0.6688 e-0.20 p \\
& \text { Dscore }=0.094 n^{1 / 2}+0.60 e-0.324 p \\
& \text { Where, } \\
& n=\text { number of site points (capped at 100) } \\
& e=\text { enclosure score, and } \\
& p=\text { hydrophilic score (capped at 1.0). }
\end{aligned}
$$

For SiteScore the hydrophilic score is capped at 1.0 whereas the hydrophilic score is uncapped in Dscore to penalize highly polar sites. This critical feature in Dscore classifies binding sites between "druggable," "difficult," and "undruggable" sites on a protein.

\section{RESULTS AND DISCUSSION}

A dataset of twenty-four different X-ray crystal structures of GSK-3 $\beta$ was prepared and submitted to SiteMap for druggability assessment, as described in the 
methods section. We denote an average of eight surface pockets found on the structure of GSK-3 $\beta$. Among them, three pockets (1, 4 and 7) were consistently retrieved in all crystal structures analyzed by SiteMap.

\section{Classifying ligand-binding sites of GSK-3ß}

We first analyzed the SiteScore data to identify plausible ligand-binding sites. Based on the previously recommended cut-off scores, SiteScore can be applied as a classifier to predict ligand-binding sites (SiteScore $\geq 0.80$ ) or non-ligandbinding sites (SiteScore < 0.80) and a score higher than 1.01 indicate highly potential binding sites ${ }^{26}$. Pockets 1 and 7 demonstrate SiteScores higher than 0.80 with promising ligand-binding capabilities, as shown in Table 1. Interestingly, for site 7 the PDB entry 1 Iog and 4NMo reveal two separate pockets very close in space surrounded by the same region. In these cases, the observed low scores were correlated with the small volume of each pocket. In several cases across our dataset, six pockets $(2,3,4,5,6$, and 8$)$ have median SiteScores less than the cut-off range.

\section{Classifying druggable binding sites of GSK-3 $\beta$}

In addition to SiteScore, we also analyzed the druggability of each site of GSK-3 $\beta$ (Table 2). Considering the Dscore criteria, binding sites of a protein can be classified into "druggable," "undruggable," and "medium druggable/difficult" sites ${ }^{26}$.

Druggable site (Dscore > o.98)

A typical druggable site is recognized by its good size, deeply buried pocket and often hydrophobic character. Among the eight sites identified, pocket 1 was the largest predicted site with a median Dscore higher than 0.98 (83\% cases). Moreover, undruggable sites (Dscore $<0.83$ ) were not identified in any cases for pocket 1 , indicating a druggable pocket of GSK-3 $\beta$ (Table 2). All other pockets, in most cases, fail the druggability criteria.

Difficult sites (Dscore between 0.83 and 0.98 )

SiteMap druggability scores recognize pocket 7 as a "difficult" site with the highest predicted cases scoring between 0.83 and 0.98 . In addition, in few cases pocket $1(16 \%)$, pocket $2(5 \%)$, pocket $3(18 \%)$, and pocket $4(13 \%)$ scored in the intermediate range. Despite the adequate size and volume of the site, with exceptional hydrophilicity, an important reason to classify site 7 as an "intermediate site" is the low hydrophobic nature of the site (quantitative median phobic score is approximately 0.3) (Table 3). To facilitate comparison, we visualized the surface maps of a "druggable" site (pocket 1) and a "difficult" site (pocket 7) identified by SiteMap (Figure 2 and 3). A clear difference is observed for pocket 7 , 
which lacks sufficient sized hydrophobic regions (yellow maps). The hydrogenbond donor and acceptor regions of this pocket (blue and red maps, respectively) are scattered over the entire cavity while these regions are more concentrated surrounding the entire hydrophobic region of pocket 1.

Furthermore, we explored the quantitative pocket characteristics of site 7 , as summarized in Table 3. This site is relatively open to solvent with moderately high average exposure scores of 0.69 (calibrated score: 0.49). Moreover, the site is partially buried with an average enclosure score of 0.64 (calibrated score: 0.78; higher scores are considered better for a deeply buried pocket). The degree of contact measures the relative tightness between a ligand and the binding site. Here, the contact property displayed relatively lower scores, observed to be 0.8 compared to the standard values (calibrated score: $\sim 1$ ). As a result, it would be more challenging to design high affinity drug-like molecules for this site. Moreover, the donor/acceptor character of this pocket quantifies moderate hydrogen-bond possibilities between a well-structured ligand and the site. These features represent the overall characteristics of a "difficult" pocket assessed by SiteMap.

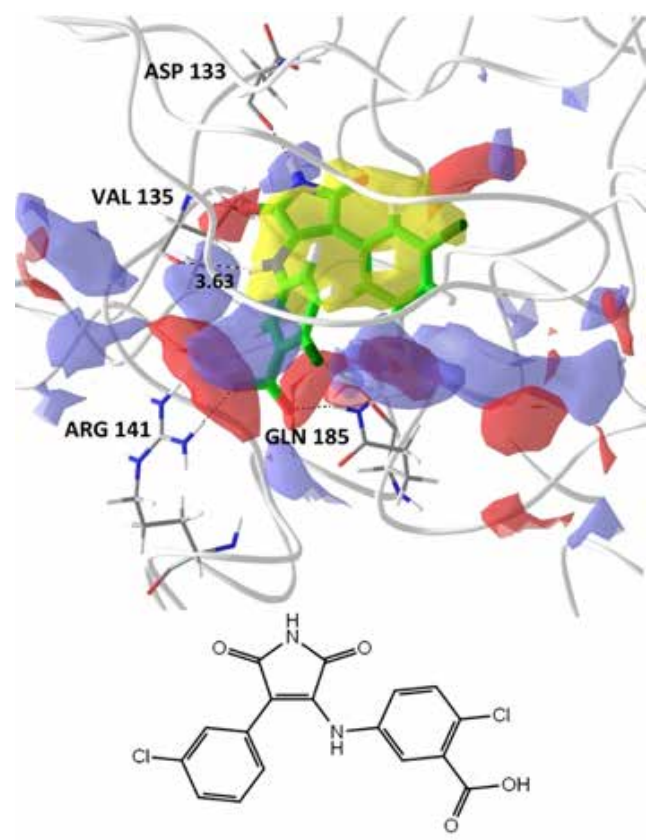

Figure 2. Hydrophobic (yellow), hydrogen-bond donor (blue), and acceptor maps (red) of site 1 (druggable pocket) with co-crystallized ligand 3-anilino-4-arylmaleimide along with a two dimensional (2D) structure representation. (PDB ID: 1Q4L represented as a thin gray tube). For clarity, site points were removed over the entire binding pocket. 


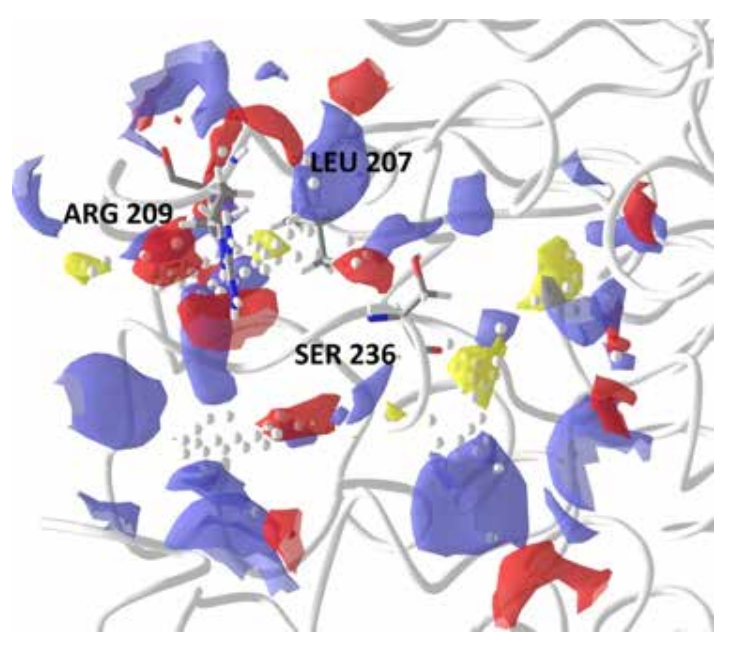

Figure 3. Hydrophobic (yellow), hydrogen-bond donor (blue), and acceptor maps (red) of site 7 (difficult pocket) identified by SiteMap. (PDB ID: 1PYX represented as a thin gray tube and the site points as white spheres).

(iii) Undruggable sites (Dscore $<0.83$ )

The undruggable sites are shallow protein surface pockets, extremely hydrophilic with negligible hydrophobic nature, characterized by Dscores below 0.80 . The druggability scores recognize pockets 5,6 , and 8 as undruggable sites (100\% cases). Several cases for pocket 2 (95\%) and pocket 4 (87\%) were also categorized as undruggable sites (Table 2). SiteMap identified pocket 3 as having two or three distinct cavities within the same binding region, annotated with their respective scores shown in Tables 1 and 2. This yielded a total of 33 sites, of which 27 sites exhibited median Dscores, less than 0.83 (82\% undruggable cases). 
Table 1. Performance in classifying binding sites of glycogen synthase kinase-3 $\beta$ based on SiteScore.

\begin{tabular}{|c|c|c|c|c|c|c|c|c|}
\hline PDB code & $\begin{array}{c}\text { Pocket } \\
1\end{array}$ & $\begin{array}{c}\text { Pocket } \\
2\end{array}$ & $\begin{array}{c}\text { Pocket } \\
3\end{array}$ & $\begin{array}{c}\text { Pocket } \\
4\end{array}$ & $\begin{array}{c}\text { Pocket } \\
5\end{array}$ & $\begin{array}{c}\text { Pocket } \\
6\end{array}$ & $\begin{array}{c}\text { Pocket } \\
7\end{array}$ & $\begin{array}{c}\text { Pocke } \\
8\end{array}$ \\
\hline $1 G N G$ & 0.891 & 0.523 & $0.726,0.690,0.553$ & 0.553 & n.f. & 0.553 & 0.963 & 0.743 \\
\hline $1 \mathrm{H} 8 \mathrm{~F}$ & 1.012 & 0.525 & n.f. & 0.720 & n.f. & 0.722 & 0.937 & 0.666 \\
\hline 1109 & 1.035 & n.f. & n.f. & 0.757 & 0.703 & 0.640 & $0.734,0.674$ & 0.712 \\
\hline $109 U$ & 0.991 & n.f. & $0.856,0.639$ & 0.718 & n.f. & $0.631,0.592$ & 0.968 & 0.722 \\
\hline $1 P Y X$ & 1.029 & 0.552 & n.f. & 0.684 & 0.636 & n.f. & 0.934 & 0.697 \\
\hline 1Q3D & 0.923 & 0.717 & 0.566 & 0.793 & 0.690 & n.f. & 0.938 & n.f. \\
\hline $103 \mathrm{~W}$ & 1.070 & n.f. & 0.574 & 0.775 & 0.652 & 0.661 & 0.944 & n.f. \\
\hline $1 Q 41$ & 1.097 & 0.742 & n.f. & 0.680 & 0.670 & 0.581 & 0.967 & n.f. \\
\hline 1Q4L & 1.045 & 0.788 & 0.588 & 0.860 & 0.621 & n.f. & 0.912 & n.f. \\
\hline $105 K$ & 1.014 & n.f. & $0.659,0.561$ & 0.681 & 0.659 & 0.494 & 0.914 & 0.709 \\
\hline 1ROE & 1.033 & n.f. & $0.773,0.568$ & 0.805 & 0.608 & 0.570 & 0.946 & 0.711 \\
\hline 1UV5 & 1.043 & $0.701,0.605$ & $0.748,0.621$ & 0.730 & 0.713 & 0.823 & 0.973 & 0.726 \\
\hline $205 K$ & 1.031 & 0.804 & 0.709 & 0.680 & n.f. & 0.745 & 0.969 & 0.664 \\
\hline 20W3 & 1.035 & 0.636 & $0.836,0.623$ & 0.919 & n.f. & n.f. & 0.957 & 0.772 \\
\hline 3DU8 & 1.058 & $0.628,0.627$ & $0.862,0.686$ & 0.746 & n.f. & n.f. & 0.947 & n.f. \\
\hline $3 F 88$ & 1.118 & 0.822 & $0.691,0.637$ & 0.940 & n.f. & 0.479 & 0.870 & 0.636 \\
\hline 3GB2 & 1.046 & 0.584 & n.f. & 0.661 & n.f. & 0.484 & 0.945 & 0.631 \\
\hline 3PUP & 1.038 & 0.597 & 0.795 & 0.816 & 0.631 & 0.596 & 0.899 & n.f. \\
\hline 3ZRM & 1.016 & 0.575 & $0.728,0.700,0.570$ & 0.645 & n.f. & n.f. & 0.962 & 0.649 \\
\hline $4 A C D$ & 1.033 & 0.674 & 0.686 & 0.742 & 0.644 & n.f. & 0.962 & n.f. \\
\hline 4J1R & 0.930 & 0.709 & 0.845 & 0.627 & n.f. & 0.636 & 0.954 & 0.668 \\
\hline $4 J 71$ & 1.034 & n.f. & 0.664 & 0.681 & 0.724 & n.f. & 0.994 & 0.664 \\
\hline 4NMO & 0.996 & n.f. & $0.867,0.626$ & 0.736 & 0.718 & n.f. & $0.754,0.680$ & 0.744 \\
\hline 4NU1 & 1.070 & 0.930 & $0.813,0.651,0.635$ & 0.735 & 0.632 & 0.627 & 0.810 & 0.766 \\
\hline Site Score $\geq 0.80$ & 24 & 03 & 06 & 05 & 00 & 01 & 22 & 00 \\
\hline Site Score $<0.80$ & 00 & 16 & 27 & 19 & 14 & 15 & 04 & 17 \\
\hline Site not found & none & 07 & 05 & none & 10 & 09 & none & 07 \\
\hline
\end{tabular}

n.f. denotes cavity was not found. 
Table 2. Performance in classifying binding sites of GSK-3 $\beta$ based on Dscore.

\begin{tabular}{|c|c|c|c|c|c|c|c|c|}
\hline PDB code & $\begin{array}{c}\text { Pocket } \\
1\end{array}$ & $\begin{array}{c}\text { Pocket } \\
2 \\
\end{array}$ & $\begin{array}{c}\text { Pocket } \\
3 \\
\end{array}$ & $\begin{array}{c}\text { Pocket } \\
4\end{array}$ & $\begin{array}{c}\text { Pocket } \\
5 \\
\end{array}$ & $\begin{array}{c}\text { Pocket } \\
6 \\
\end{array}$ & $\begin{array}{c}\text { Pocket } \\
7\end{array}$ & $\begin{array}{c}\text { Pocket } \\
8 \\
\end{array}$ \\
\hline $1 G N G$ & 0.868 & 0.430 & $0.680,0.678,0.362$ & 0.461 & n.f. & 0.484 & 0.974 & 0.720 \\
\hline $1 \mathrm{H} 8 \mathrm{~F}$ & 1.002 & 0.342 & n.f. & 0.665 & n.f. & 0.638 & 0.943 & 0.615 \\
\hline 1109 & 1.005 & n.f. & n.f. & 0.713 & 0.575 & 0.596 & $0.661,0.620$ & 0.678 \\
\hline $109 \mathrm{U}$ & 1.013 & n.f. & $0.904,0.418$ & 0.590 & n.f. & $0.568,0.549$ & 0.994 & 0.641 \\
\hline $1 \mathrm{PYX}$ & 0.987 & 0.488 & n.f. & 0.612 & 0.567 & n.f. & 0.953 & 0.671 \\
\hline $1 Q 3 D$ & 0.896 & 0.576 & 0.487 & 0.769 & 0.647 & n.f. & 0.953 & n.f. \\
\hline $103 \mathrm{~W}$ & 1.054 & n.f. & 0.505 & 0.732 & 0.623 & 0.655 & 0.960 & n.f. \\
\hline $1 Q 41$ & 1.114 & 0.723 & n.f. & 0.622 & 0.643 & 0.532 & 0.999 & n.f. \\
\hline $1 Q 4 \mathrm{~L}$ & 1.068 & 0.752 & 0.548 & 0.868 & 0.542 & n.f. & 0.930 & n.f. \\
\hline $105 \mathrm{~K}$ & 1.023 & n.f. & $0.649,0.516$ & 0.619 & 0.602 & 0.410 & 0.924 & 0.233 \\
\hline 1ROE & 1.057 & n.f. & $0.809,0.516$ & 0.742 & 0.564 & 0.381 & 0.957 & 0.653 \\
\hline 1UV5 & 0.969 & $0.516,0.553$ & $0.747,0.570$ & 0.670 & 0.652 & 0.776 & 0.936 & 0.691 \\
\hline $205 \mathrm{~K}$ & 1.003 & 0.690 & 0.588 & 0.552 & n.f. & 0.732 & 0.979 & 0.628 \\
\hline 20W3 & 1.073 & 0.599 & $0.851,0.466$ & 0.935 & n.f. & n.f. & 0.976 & 0.721 \\
\hline 3DU8 & 1.058 & $0.571,0.573$ & $0.894,0.673$ & 0.725 & n.f. & n.f. & 0.946 & n.f. \\
\hline $3 F 88$ & 1.133 & 0.800 & $0.555,0.604$ & 0.989 & n.f. & 0.359 & 0.874 & 0.573 \\
\hline 3GB2 & 1.053 & 0.520 & n.f. & 0.603 & n.f. & 0.405 & 0.968 & 0.595 \\
\hline 3PUP & 1.065 & 0.475 & 0.820 & 0.787 & 0.574 & 0.560 & 0.904 & n.f. \\
\hline 3ZRM & 1.026 & 0.454 & $0.696,0.686,0.537$ & 0.596 & n.f. & n.f. & 0.986 & 0.598 \\
\hline $4 A C D$ & 1.038 & 0.624 & 0.590 & 0.696 & 0.601 & n.f. & 0.983 & n.f. \\
\hline $4 J 1 R$ & 0.933 & 0.566 & 0.854 & 0.579 & n.f. & 0.509 & 0.982 & 0.617 \\
\hline $4 \mathrm{~J} 71$ & 1.057 & n.f. & 0.543 & 0.637 & 0.691 & n.f. & 1.031 & 0.598 \\
\hline 4NMO & 0.982 & n.f. & $0.928,0.496$ & 0.715 & 0.650 & n.f. & $0.728,0.510$ & 0.728 \\
\hline 4NU1 & 1.083 & 0.882 & $0.857,0.469,0.561$ & 0.703 & 0.573 & 0.499 & 0.730 & 0.731 \\
\hline $\begin{array}{l}\text { Dscore }>0.98 \\
\text { (druggable) }\end{array}$ & 20 & 00 & 00 & 00 & 00 & 00 & 06 & 00 \\
\hline $\begin{array}{l}\text { Dscore (0.83-0.98) } \\
\text { (difficult) }\end{array}$ & 04 & 01 & 06 & 03 & 00 & 00 & 15 & 00 \\
\hline $\begin{array}{l}\text { Dscore }<0.83 \\
\text { (undruggable) }\end{array}$ & 00 & 18 & 27 & 21 & 14 & 16 & 05 & 17 \\
\hline
\end{tabular}

n.f. denotes cavity was not found. 
Table 3. SiteMap property values of pocket 7 (Allosteric site) of GSK-3ß.

\begin{tabular}{|c|c|c|c|c|c|c|c|c|c|}
\hline PDB code & Size & Volume & Exposure & Enclosure & Contact & Phobic & Philic & Balance & $\begin{array}{c}\text { Donor/ } \\
\text { acceptor ratio }\end{array}$ \\
\hline $1 \mathrm{GNG}$ & 111 & 312.816 & 0.671 & 0.643 & 0.799 & 0.280 & 1.079 & 0.260 & 1.154 \\
\hline $1 \mathrm{H} 8 \mathrm{~F}$ & 94 & 284.690 & 0.717 & 0.637 & 0.808 & 0.286 & 1.076 & 0.265 & 1.034 \\
\hline \multirow{2}{*}{1109} & 50 & 143.717 & 0.717 & 0.621 & 0.859 & 0.179 & 1.154 & 0.155 & 0.771 \\
\hline & 33 & 128.968 & 0.783 & 0.652 & 0.810 & 0.338 & 0.953 & 0.355 & 1.185 \\
\hline $109 \mathrm{U}$ & 105 & 320.362 & 0.693 & 0.643 & 0.803 & 0.359 & 1.017 & 0.353 & 1.093 \\
\hline $1 \mathrm{PYX}$ & 91 & 279.888 & 0.709 & 0.638 & 0.812 & 0.353 & 0.001 & 0.353 & 0.969 \\
\hline $1 Q 3 D$ & 95 & 277.830 & 0.716 & 0.633 & 0.824 & 0.252 & 1.052 & 0.240 & 1.123 \\
\hline $103 W$ & 92 & 260.337 & 0.690 & 0.655 & 0.880 & 0.395 & 1.026 & 0.385 & 0.922 \\
\hline $1 Q 41$ & 119 & 298.753 & 0.654 & 0.630 & 0.820 & 0.377 & 0.978 & 0.386 & 0.835 \\
\hline $1 Q 4 \mathrm{~L}$ & 84 & 290.864 & 0.728 & 0.631 & 0.823 & 0.443 & 0.951 & 0.466 & 1.069 \\
\hline $105 \mathrm{~K}$ & 86 & 290.178 & 0.730 & 0.643 & 0.798 & 0.410 & 1.021 & 0.402 & 1.220 \\
\hline 1ROE & 97 & 300.468 & 0.731 & 0.634 & 0.819 & 0.327 & 1.071 & 0.305 & 1.145 \\
\hline 1UV5 & 111 & 312.473 & 0.683 & 0.658 & 0.875 & 0.131 & 1.222 & 0.108 & 0.780 \\
\hline $205 \mathrm{~K}$ & 120 & 303.555 & 0.636 & 0.652 & 0.844 & 0.476 & 1.081 & 0.440 & 0.832 \\
\hline 20W3 & 116 & 319.333 & 0.707 & 0.634 & 0.818 & 0.231 & 1.055 & 0.219 & 1.315 \\
\hline 3DU8 & 93 & 286.405 & 0.677 & 0.658 & 0.864 & 0.351 & 1.090 & 0.322 & 1.114 \\
\hline $3 F 88$ & 77 & 287.091 & 0.779 & 0.626 & 0.738 & 0.140 & 1.000 & 0.140 & 1.128 \\
\hline $3 G B 2$ & 97 & 257.250 & 0.709 & 0.626 & 0.805 & 0.347 & 1.022 & 0.340 & 1.191 \\
\hline 3PUP & 84 & 223.979 & 0.679 & 0.638 & 0.790 & 0.269 & 1.046 & 0.257 & 1.427 \\
\hline 3ZRM & 102 & 301.154 & 0.690 & 0.640 & 0.801 & 0.459 & 1.038 & 0.443 & 1.026 \\
\hline $4 A C D$ & 108 & 297.038 & 0.667 & 0.641 & 0.828 & 0.424 & 1.047 & 0.405 & 1.122 \\
\hline $4 J 1 R$ & 112 & 286.748 & 0.652 & 0.623 & 0.803 & 0.370 & 1.017 & 0.364 & 1.065 \\
\hline $4 J 71$ & 104 & 281.260 & 0.633 & 0.659 & 0.859 & 0.536 & 0.935 & 0.573 & 1.220 \\
\hline \multirow{2}{*}{ 4NM0 } & 40 & 133.427 & 0.688 & 0.671 & 0.818 & 0.727 & 0.826 & 0.879 & 1.293 \\
\hline & 38 & 84.721 & 0.568 & 0.639 & 0.849 & 0.000 & 1.389 & 0.000 & 1.227 \\
\hline 4NU1 & 59 & 186.249 & 0.704 & 0.668 & 0.856 & 0.117 & 1.205 & 0.097 & 0.619 \\
\hline Average value & 89 & 259.598 & 0.693 & 0.642 & 0.823 & 0.330 & 1.014 & 0.327 & 1.072 \\
\hline
\end{tabular}

Phobic and philic terms are the hydrophobic and hydrophilic scores, respectively.

\section{Computational Validation of Generated Pockets}

The pockets identified by SiteMap analysis were validated with the known cocrystal structures available at the time of the study. Seven different cavities are supported by X-ray crystallographic studies in the Protein Data Bank (PDB) where ligands (pocket 1), peptides (pocket 2 and 3 ) and even heteroatoms (pocket $4,5,7$, and 8) are known to be captured within these cavities, while pocket 6 
represents an orphan site.

Pocket 1: A variety of heterocyclic ligands are known to mimic the GSK-3 $\beta$ active site. To validate this site, the PDB structure of $1 \mathrm{Q} 4 \mathrm{~L}$ was selected which has an anilino-maleimide crystallized within GSK- $3 \beta^{27}$. Quesada-Romero et al. reported the orientation of several maleimide derivatives that adopt the GSK-3 $\beta$ active site $^{28}$. Figure 2 has been taken from the same perspective to confirm the druggability and binding of such ligands to this pocket. A maleimide core structure attached to two aryl rings occupies the yellow hydrophobic region. The carboxylate group and one of the acyl groups of the ligand lie in the red acceptor region, while the $\mathrm{NH}$ group of the core maleimide structure occupies the blue donor region. The anilino group just failed to spot the donor region. Here, SiteMap accuracy is judged as the distance between the anilino group and the carbonyl oxygen atom of Val135 is $3.63 \AA$, which is quiet far for a strong hydrogen-bond interaction.

Pocket 2: This pocket is recognized as the substrate binding site. A pS9 autoinhibitory peptide ${ }^{29}$ recognizing the substrate site is shown in figure 4. Key hydrogen-bond interactions are recognized with the primed phosphate groups of the peptide and a triad of three basic residues (Arg96, Arg180, and Lys205). Furthermore, the backbone of the peptide is shown to interact with the Lys94 residue of this site. These interactions confirm the binding of such peptides in this pocket.
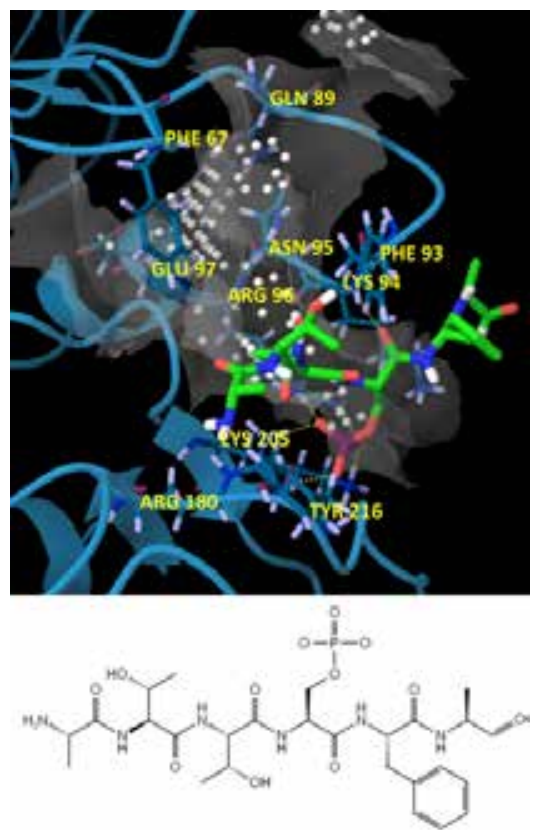

Figure 4. A pS9 auto-inhibitory peptide is shown to interact with the substrate site of GSK$3 \beta$ in the 4NU1 structure together with a 2D structure representation. 
Pocket 3: The Axin and Fratide peptides recognize the peptide-binding channel comprising $\alpha$-helix (residues 262-273) and the extended loop (residues 285299 ) at the C-lobe of GSK- $33^{30-32}$. In several cases across our dataset, two to three small cavities were observed within the entire channel. Among these, a distinct "hydrophobic patch" identified by SiteMap formed with the hydrophobic residues of the $\alpha$-helix, and the extended loop provides favorable peptidebinding characteristics (Figure 5).

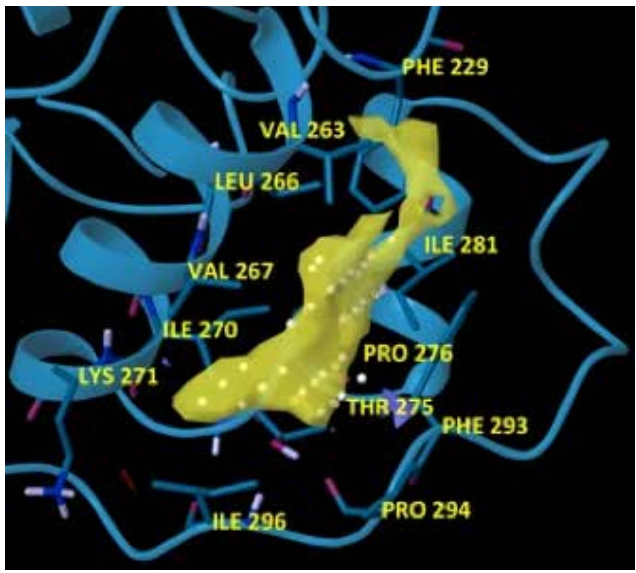

Figure 5. Pocket 3 identified as a hydrophobic patch by SiteMap within the peptide-binding channel at the C-lobe of GSK-3ß.

Key hydrogen-bond interactions are recognized with residues Tyr288 and Glu290 and the Fratide peptide (Figure 6B) and with the Asp264 residue and the Axin peptide (Figure 6A).
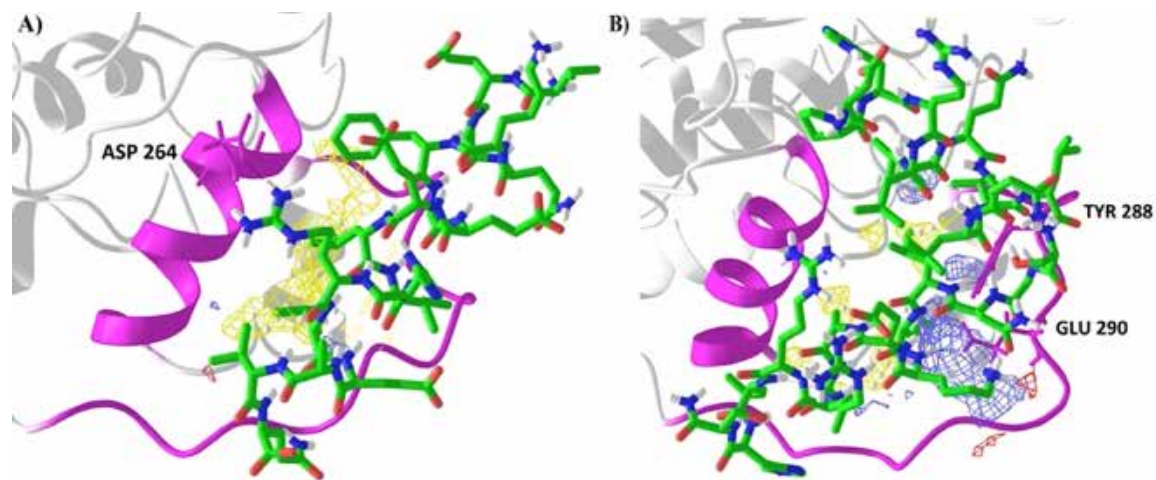

Figure 6. A) Axin peptide represented as green tube recognize the peptide-binding channel (purple cartoon representation) with key $\mathrm{H}$-bond interaction represented as black dots (PDB ID: 109U). B) Fratide peptide represented as green tube recognize the peptide-binding channel with key H-bond interactions represented as black dots (PDB ID: 1GNG). 
Pocket 4: This pocket was visible in each case analyzed by SiteMap. The cavity generated by SiteMap and the presence of glycerol with a hydrogen bond to the Arg144 residue is observed in the PDB structure of 4NU1 shown in Figure 7. Key interactions are observed between the oxygen atom of the glycerol molecule and the guanidine NH group of Arg144.

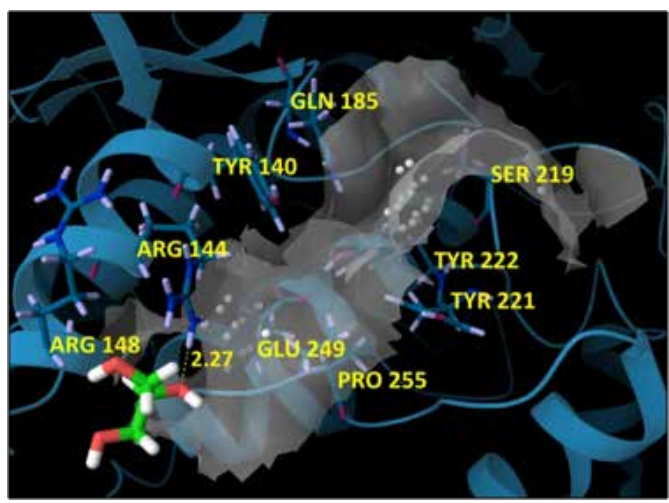

Figure 7. A glycerol molecule represented as a green tube is shown to capture the surface of pocket 4 (gray) with the key H-bond highlighted as yellow dots (PDB ID: 4NU1, blue cartoon representation).

Pocket 5: A shallow pocket is located at the N-lobe of GSK-3 $\beta$. In the PDB structure of $4 \mathrm{NU1}$, a molecule of glycerol is shown to bind in this pocket. The polar and charged residues (Tyr56, Lys86, and Asn129) surrounding the small cavity are significant enough to form hydrogen-bond contacts with small hydrophilic glycerol molecules, as shown in Figure 8.

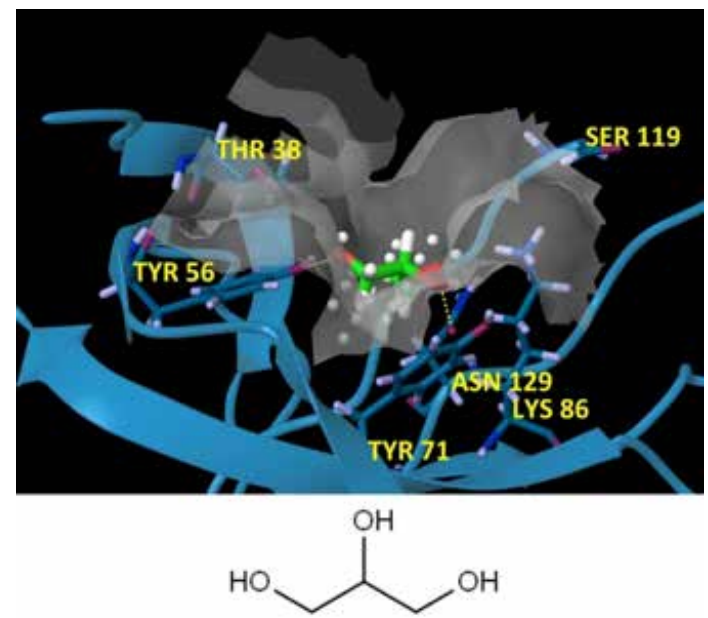

Figure 8. A glycerol molecule represented as a green tube together with a $2 \mathrm{D}$ structure is shown to capture the surface of pocket 5 (gray) with the key H-bond highlighted as yellow dots (PDB ID: 4NU1, blue cartoon representation). 
Pocket 6: The major residues lining a small cavity in the hinge domain were identified as pocket 6 (Figure 9). No ligands or heteroatoms are known to bind in this pocket, which represents an orphan site.

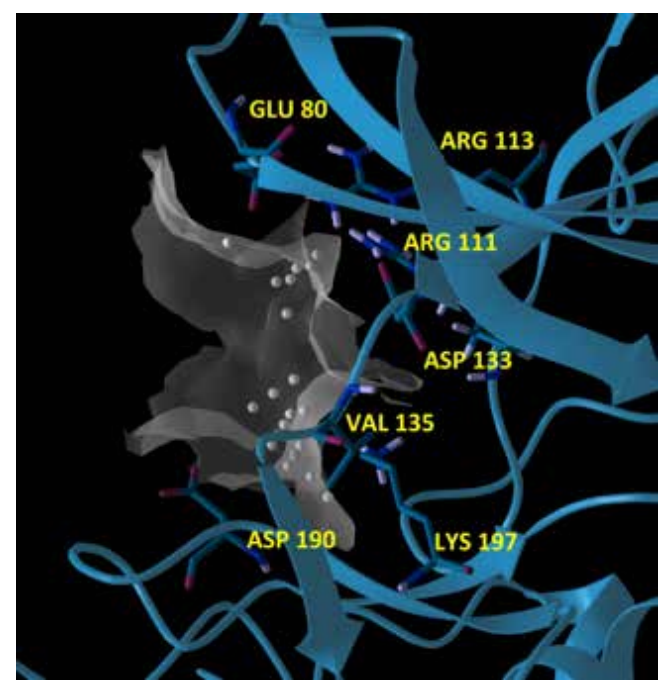

Figure 9. The total surface of pocket 6 represented as gray and the site points as white spheres (PDB ID: 109U, blue cartoon representation).

Pocket 7: SiteMap scores recognize pocket 7 as the most promising allosteric site of GSK-3 $\beta$. In the crystallization experiments, a few heteroatom and reagent molecules are known to be captured in this pocket. To support our result, the PDB structure of 4 NMo was selected, which has Dithiothreitol (DTT) molecule crystallized within GSK- $3 \beta^{29}$. Key hydrogen-bond interaction is recognized between the DTT molecule and residues Thr326, Ala327, and Arg319 of pocket 7, as shown in Figure 10. In addition, the binding residues Arg209 and His173 of the same pocket in the PDB structures of $1 \mathrm{UV}_{5}$, 1Io9, and 1GNG also chelates phosphate and sulfate ions present in the crystallization experiments. These interactions are crucial for understanding the important residues for allosteric modulation of the kinase.

Pocket 8: A new small pocket identified by SiteMap is located at the C-lobe of GSK-3 $\beta$. The presence of glycerol with a hydrogen-bond to Val155 is observed in the PDB structures of 3ZRM (Figure 11). The presence of such hydrophilic molecules can provide clues for the prevalence of these pockets on GSK-3 $\beta$. 

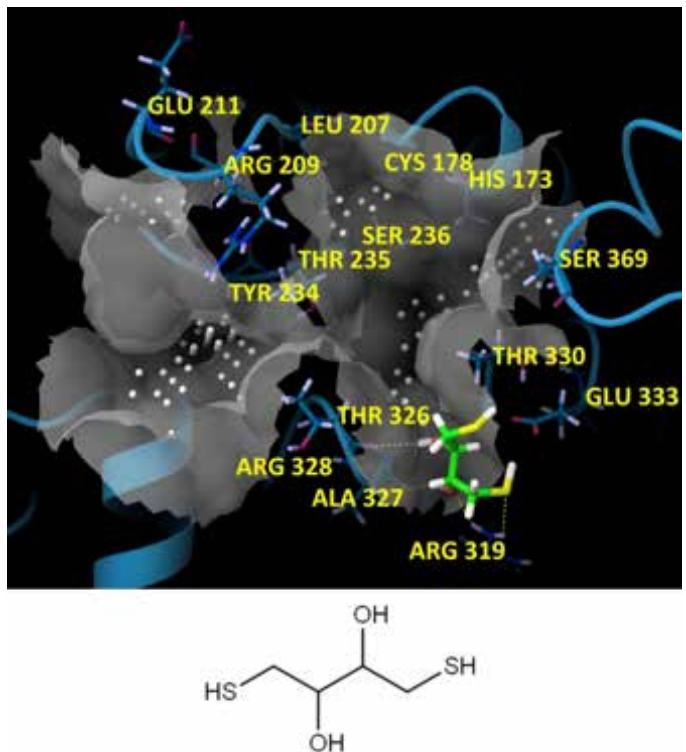

Figure 10. A Dithiothreitol (DTT) molecule represented as a green tube together with a 2D structure is shown to capture the surface of pocket 7 (gray), with the key $\mathrm{H}$-bond highlighted as yellow dots (PDB ID: 4NM0, blue cartoon representation).

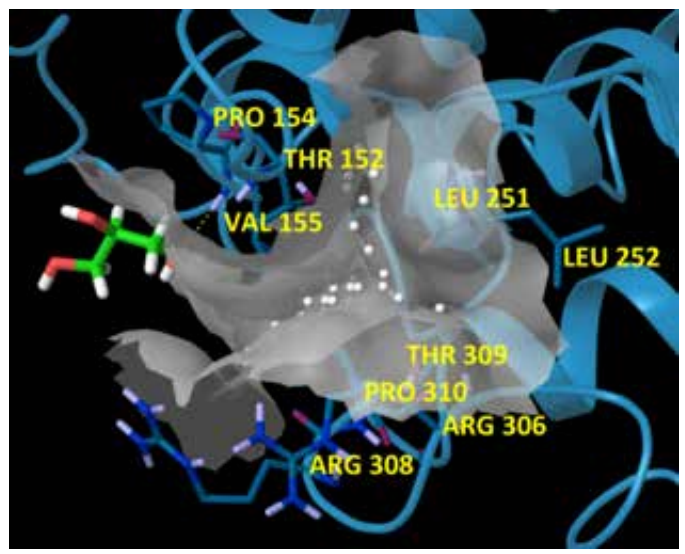

Figure 11. A glycerol molecule represented as a green tube is shown to capture the surface of pocket 8 (gray) with the key H-bond highlighted as yellow dots (PDB ID: 3ZRM, blue cartoon representation).

\section{CONCLUSIONS}

Predicted druggability on the pockets of GSK- $3 \beta$ were assessed by SiteMap. With the aim to identify druggable sites of GSK- $3 \beta$, SiteMap studies yielded useful insights that clearly distinguish druggable, difficult, and undruggable sites. These results conclude that the ATP-binding site is the only druggable pocket of GSK- 
$3 \beta$, while pocket 7 is a "difficult" pocket, as analyzed by SiteMap. Although this pocket is classified as a "difficult site" by SiteMap analysis, it has earmarks as a good binding site and the possibility of designing selective allosteric modulators. With the help of SiteScore we successfully differentiated the ligand and non-ligand-binding sites of GSK-3 $\beta$; however, the identification of non-ligandbinding sites or difficult/undruggable sites does not mean that a ligand cannot bind to such sites. Moreover, it would be challenging to search such drug-like ligands that bind with high affinity on these sites. At the moment, SiteMap studies have classified the pockets of GSK- $3 \beta$ based on the druggability score, which can clearly classify these sites between druggable, difficult, and undruggable sites. We predict that these results will add to the accuracy in identifying druggable pockets of GSK-3ß.

\section{ACKNOWLEDGEMENTS}

We thank Schrödinger LLC for the generous supply of the software used for this research.

\section{CONFLICT OF INTEREST}

None declared.

\section{REFERENCES}

1. Woodgett, J.R. Molecular cloning and expression of glycogen synthase kinase-3/factor A. EMBOJ. 1990, 9, 2431-2438.

2. Liang, M.H.; Chuang, D.M. Regulation and function of glycogen synthase kinase-3 isoforms in neuronal survival. J. Biol. Chem. 2007, 282, 3904-3917.

3. Cormier, K.W.; Woodgett J.R. Recent advances in understanding the cellular roles of GSK-3. F1oooResearch 2017, 6(F10oo Faculty Rev):167.

4. Mukai, F.; Ishiguro, K.; Sano, Y.; Fujita, S.C. Alternative splicing isoform of tau protein kinase I/glycogen synthase kinase $3 \beta . J$. Neurochem. 2002, 81, 1073-1083.

5. Hooper, C.; Killick, R.; Lovestone, S. The GSK3 hypothesis of Alzheimer's disease. J. Neurochem. 2008, 104, 1433-1439.

6. Prati, F.; Cavalli, A.; Bolognesi, M.L. Navigating the Chemical Space of Multitarget-Directed Ligands: From Hybrids to Fragments in Alzheimer's Disease. Molecules 2016, 21, 466.

7. Cai, Z.; Zhao, Y.; Zhao, B. Roles of glycogen synthase kinase 3 in Alzheimer's disease. Curr. Alzheimer Res. 2012, 9, 864-879.

8. Llorens-Marítin, M.; Jurado, J.; Hernández, F.; Ávila, J. GSK-3beta, a pivotal kinase in Alzheimer disease. Front. Mol. Neurosci. 2014, 7, 46.

9. Li, X.; Liu, M.; Cai, Z.; Wang, G.; Li, X. Regulation of glycogen synthase kinase-3 during bipolar mania treatment. Bipolar Disord. 2o10, 12, 741-752.

10. Jope, R.S.; Roh, M.S. Glycogen synthase kinase-3 (GSK3) in psychiatric diseases and therapeutic interventions. Curr. Drug Targets 2006, 7, 1421-1434. 
11. Manoukian, A.S.; Woodgett, J.R. Role of glycogen synthase kinase-3 in cancer: regulation by Wnts and other signaling pathways. Adv. Cancer Res. 2002, 84, 203-229.

12. Piazza, F.; Manni, S.; Semenzato, G. Novel players in multiple myeloma pathogenesis: role of protein kinases CK2 and GSK3. Leukemia Res. 2013, 37, 221-227.

13. Nikoulina, S.E.; Ciaraldi, T.P.; Mudaliar, S.; Mohideen, P.; Carter L.; Henry, R.R. Potential role of glycogen synthase kinase- 3 in skeletal muscle insulin resistance of type 2 diabetes. Diabetes 2000, 49, 263-271.

14. Martinez, A.; Castro, A.; Dorronsoro, I.; Alonso, M. Glycogen synthase kinase 3 (GSK-3) inhibitors as new promising drugs for diabetes, neurodegeneration, cancer, and inflammation. Med. Res. Rev. 2002, 22, 373-384.

15. Cohen, P.; Goedert, M. GSK3 inhibitors: development and therapeutic potential. Nat. Rev. Drug Discov. 2004, 3, 479-487.

16. Jope, R.S.; Yuskaitis, C.J.; Beurel, E. Glycogen synthase kinase-3 (GSK3): inflammation, diseases, and therapeutics. Neurochem Res. 2007, 32, 577-595.

17. Beurel, E.; Grieco, S.F.; Jope, R.S. Glycogen synthase kinase-3 (GSK3): regulation, actions, and diseases. Pharmacol. Ther. 2015, 148, 114-131.

18. Eldar-Finkelman, H.; Licht-Murava, A.; Pietrokovski, S.; Eisenstein, M. Substrate competitive GSK-3 inhibitors - strategy and implications. Biochim. Biophys. Acta 2010, 1804, 598-603.

19. Halgren, T. New method for fast and accurate binding-site identification and analysis. Chem. Biol. Drug Des. 2007, 69, 146-148.

20. Wagner, J.R.; Lee, C.T.; Durrant, J.D. Malmstrom, R.D.; Feher, V.A.; Amaro, R.E. Emerging computational methods for the rational discovery of allosteric drugs. Chem. Rev. 2016, 116, 6370-6390.

21. Palomo, V.; Soteras, I.; Perez, D.I.; Perez, C.; Gil, C.; Campillo, N.E.; Martinez, A. Exploring the binding sites of glycogen synthase kinase 3 . Identification and characterization of allosteric modulation cavities. J. Med. Chem. 2011, 54, 8461-8470.

22. ter Haar, E.; Coll, J.T.; Austen, D.A.; Hsiao, H.M.; Swenson, L.; Jain, J. Structure of GSK3beta reveals a primed phosphorylation mechanism. Nat. Struct. Biol. 2oo1, 8, 593-596.

23. Chauhan, N.; Gajjar, A.; Basha, S.H. Pharmacophore feature-based virtual screening for finding potent GSK-3 inhibitors using molecular docking and dynamics simulations. Bioinformation 2016, 12, 391-395.

24. Natarajan, P.; Priyadarshini, V.; Pradhan, D.; Manne, M.; Swargam, S.; Kanipakam, H.; Bhuma V.; Amineni U. E-pharmacophore-based virtual screening to identify GSK-3 $\beta$ inhibitors. J. Recept. Signal Transduct. 2016, 36, 445-458.

25. Arfeen, M.; Bharatam, P.V. Design of glycogen synthase kinase-3 inhibitors: an overview on recent advancements. Curr. Pharm. Des. 2013, 19, 4755-4775.

26. Halgren, T.A. Identifying and characterizing binding sites and assessing druggability. $J$. Chem. Inf. Model. 2009, 49, 377-389.

27. Bertrand, J.A.; Thieffine, S.; Vulpetti, A.; Cristiani, C.; Valsasina, B.; Knapp, S.; Kalisz, H.M.; Flocco, M. Structural characterization of the GSK-3beta active site using selective and non-selective ATP-mimetic inhibitors. J. Mol. Biol. 2oo3, 333, 393-407. 
28. Quesada-Romero, L.; Mena-Ulecia, K.; Tiznado, W.; Caballero, J. Insights into the Interactions between Maleimide Derivates and GSK $3 \beta$ Combining Molecular Docking and QSAR. PLoS One 2014, 9(7): e102212.

29. Stamos, J.L.; Chu, M.L.; Enos, M.D.; Shah, N.; Weis, W.I. Structural basis of GSK-3 inhibition by N-terminal phosphorylation and by the Wnt receptor LRP6. Elife 2014, 3, e01998.

30. Fraser, E.; Young, N.; Dajani, R.; Franca-Koh, J.; Ryves, J.; Williams, R.S.; Yeo, M.; Webster, M.T.; Richardson, C.; Smalley, M.J.; Pearl, L.H.; Harwood, A.; Dale, T.C. Identification of the Axin and Frat binding region of glycogen synthase kinase-3. J. Biol. Chem. 2oo2, 277, 2176-2185.

31. Bax, B.; Carter, P.S.; Lewis, C.; Guy, A.R.; Bridges, A.; Tanner, R.; Pettman, G.; Mannix, C.; Culbert, A.A.; Brown, M.J.; Smith, D.G.; Reith, A.D. The structure of phosphorylated GSK-3beta complexed with a peptide, FRATtide, that inhibits beta-catenin phosphorylation, Structure 2001, 9, 1143-1152.

32. Dajani, R.; Fraser, E.; Roe, S.M.; Yeo, M.; Good, V.M.; Thompson, V.; Dale, T.C.; Pearl, L.H. Structural basis for recruitment of glycogen synthase kinase 3 beta to the axin-APC scaffold complex. EMBO J. 2003, 22, 494-501.

(Received 10 April 2017; Accepted 26 April 2017) 